\title{
Static and Dynamic Components of the Redshift
}

\author{
Rajendra P. Gupta ${ }^{1,2}$ \\ ${ }^{1}$ Macronix Research Corporation, Ottawa, Canada \\ ${ }^{2}$ National Research Council, Ottawa, Canada (Retired) \\ Email: sonice810@gmail.com
}

How to cite this paper: Gupta, R.P. (2018) Static and Dynamic Components of the Redshift. International Journal of Astronomy and Astrophysics, 8, 219-229. https://doi.org/10.4236/ijaa.2018.83016

Received: April 18, 2018

Accepted: July 16, 2018

Published: August 8, 2018

Copyright $\odot 2018$ by author and Scientific Research Publishing Inc. This work is licensed under the Creative Commons Attribution International License (CC BY 4.0).

http://creativecommons.org/licenses/by/4.0/ (c) (i) Open Access

\begin{abstract}
We analyse the possibility that the observed cosmological redshift may be cumulatively due to the expansion of the universe and the tired light phenomenon. Since the source of both the redshifts is the same, they both independently relate to the same proper distance of the light source. Using this approach we have developed a hybrid model combining the Einstein de Sitter model and the tired light model that yields a slightly better fit to Supernovae Ia redshift data using one parameter than the standard $\Lambda \mathrm{CDM}$ model with two parameters. We have shown that the ratio of tired light component to the Einstein de Sitter component of redshift has evolved from 2.5 in the past, corresponding to redshift 1000 , to its present value of 1.5 . The hybrid model yields Hubble constant $H_{0}=69.11( \pm 0.53) \mathrm{km} \cdot \mathrm{s}^{-1} \cdot \mathrm{Mpc}^{-1}$ and the deceleration parameter $q_{0}=-0.4$. The component of Hubble constant responsible for expansion of the universe is $40 \%$ of $H_{0}$ and for the tired light is $60 \%$ of $H_{0}$. Consequently, the critical density is only $16 \%$ of its currently accepted value; a lot less dark matter is needed to make up the critical density. In addition, the best data fit yields the cosmological constant density parameter $\Omega_{\Lambda}=0$. The tired light effect may thus be considered equivalent to the cosmological constant in the hybrid model.
\end{abstract}

\section{Keywords}

Galaxies: Distances and Redshifts, Cosmological Parameters, Cosmic Background Radiation, Distance Scale, Cosmology Theory

\section{Introduction}

Until the discovery of cosmic microwave background radiation by Penzias and Wilson in 1964 [1], there was a debate about the cause of the redshift of light from extragalactic objects. Since then expansion of the universe by the Big-Bang 
theory became more and more favoured explanation of the cause of the redshift rather than the tired light and steady state theories. Ironically, it is the close analysis of the cosmic microwave background that has put into question the cause of the redshift due to the discrepancy in the Hubble constant derived from the spectral data and the microwave background data [2] [3].

The Hubble constant that relates the redshift to the distance of the source of light has shown steady decline in its value from about $500 \mathrm{~km} \cdot \mathrm{s}^{-1} \cdot \mathrm{Mpc}^{-1}$ to the currently accepted value of about $69 \mathrm{~km} \cdot \mathrm{s}^{-1} \cdot \mathrm{Mpc}^{-1}$ with improvements in measuring techniques and availability of redshift-distance data for high redshift sources. However, many cosmological models seek even lower value of the Hubble constant. Since the value of the Hubble constant determines plethora of cosmological parameters, including age, size and critical density of the universe, it is important to re-examine the potential causes of the redshift, or perhaps the composition of the redshift.

The status of the expanding universe and steady state theories has been recently reviewed by López-Corredoira [4] and Orlov and Raikov [5]. They concluded that based on the currently available observational data it is not possible to unambiguously identify the preferred approach to cosmology.

In a recent paper it was shown phenomenologically that Mach effect may be the cause of tired light redshift and may contribute dominantly to the cosmological redshift [6]. While the paper's assumption that observed redshift may be the hybrid of the expansion of the universe and tired light effect may be sound, it incorrectly divided the distance modulus between the two components rather than keeping the proper distance the same and dividing the redshift.

Using Poisson's work on the motion of point particles in curved spacetime [7], Fischer [8] has shown analytically that gravitational back reaction may be responsible for the tired light phenomenon and could account for some or most of the observed redshift. His finding may also be related to Mach effect.

Tired light, hereafter Mach effect, redshift approach defines the distance $d$ of the light emitting source, whose redshift is $z$, by the equation:

$$
d=R_{0} \ln (1+z),
$$

where $R_{0}=c / H_{0}$ with $\mathrm{c}$ as the speed of light and $H_{0}$ as the Hubble constant. The same distance in the standard $\Lambda \mathrm{CDM}$ model is defined as [9]:

$$
d=R_{0} \int_{0}^{z}\left[\mathrm{~d} u / \sqrt{\left(\Omega_{m, 0}(1+u)^{3}+\Omega_{\Lambda, 0}\right)}\right],
$$

where $\Omega_{m, 0}$ is the matter energy density relative to the critical energy density, $\Omega_{\Lambda, 0}$ is the relative energy density corresponding to the Einstein cosmological constant, and $\Omega_{m, 0}+\Omega_{\Lambda, 0}=1$.

Distance $d$ is determined from the measurement of the bolometric flux $f$ of the light emitting source and comparing it with the standard for that type of source with known luminosity $L$. The luminosity distance $d_{L}$ is defined as:

$$
d_{L}=\sqrt{L / 4 \pi f} .
$$


while the flux could normally be related to the luminosity $L$ with an inverse square law $f=L /\left(4 \pi d^{2}\right)$ in a flat universe, it needs to be modified to include the effects of the redshift, the expansion of the universe, and any other unknown phenomenon, in order to determine the distance $d$ of the source correctly.

\section{Theory}

If we assume the observed redshift to be due to two effects, we need to find out that in what proportion two effects contribute to the observed redshift. Let $z_{X}$ be the redshift due to expansion of the universe and $z_{M}$ be the redshift due to the Mach effect-we will use subscript $X$ for expansion of the universe and $M$ for Mach effect. The expansion will cause the emission wavelength $\lambda_{e}$ to stretch to wavelength $\lambda_{X}$ such that $\lambda_{X}=\left(1+z_{X}\right) \lambda_{e}$. This wavelength will be further increased due to the Mach effect before it is observed as wavelength

$\lambda_{0}=\left(1+z_{M}\right) \lambda_{X}=\left(1+z_{M}\right)\left(1+z_{X}\right) \lambda_{e}$. But we can also write $\lambda_{0}=(1+z) \lambda_{e} . \mathrm{We}$ may thus write:

$$
1+z=\left(1+z_{X}\right)\left(1+z_{M}\right) .
$$

Considering now the scale factor $a(t)$ relating the proper distance $d$ to the comoving distance $r$ between the galaxies, it can only depend on the component of the redshift that is due to the expansion of the universe rather than the whole redshift; i.e. $a_{X}(t)=1 /\left(1+z_{X}\right)$ should be used as the scale factor.

Let us estimate the loss of the flux of light in the measurement of the luminosity of an object due to:

1) Expansion of the universe:

a) Stretching of the wavelength $\propto 1 /\left(1+z_{X}\right)$, and

b) Increase in time between photon detection $\propto a_{X}(t)=1 /\left(1+z_{X}\right)$.

Thus the flux is reduced to $f_{X}=f /\left(1+z_{X}\right)^{2}$.

2) Mach effect: It is only due to the increase in wavelength $\propto 1 /\left(1+z_{M}\right)$.

And the flux is reduced to $f_{M}=f_{X} /\left(1+z_{M}\right)$.

3) Unknown factor: We found that there is an additional flux loss $\propto 1 / \sqrt{1+z}$. The measured bolometric flux may thus be written as:

$$
f_{B}=L /\left[4 \pi d^{2}\left(1+z_{X}\right)^{2}\left(1+z_{M}\right) \sqrt{1+z}\right] .
$$

The luminosity distance may now be written as:

$$
\begin{aligned}
d_{L} & =d\left(1+z_{X}\right)\left(1+z_{M}\right)^{\frac{1}{2}}(1+z)^{\frac{1}{4}} \\
& =d\left(1+z_{X}\right)^{\frac{1}{2}}(1+z)^{\frac{1}{2}}(1+z)^{\frac{1}{4}}
\end{aligned}
$$

Since the proper distance $d$, often written as $d_{P}(t)$, determined using either of the above models must be the same, equating them can give relationship between $z_{X}$ and $z_{M}$, and also determine the deceleration parameter $q_{0}$ in the limit of $z \ll 1$. We may rewrite Equation (1) for Mach effect as:

$$
d_{M}=R_{M} \ln \left(1+z_{M}\right) .
$$

Here $R_{M}=c / H_{M}$ with $H_{M}$ being the Hubble constant corresponding to 
the Mach effect. Regarding Equation (2) for the expansion model, we will be exploring how the density parameters, taken usually as $\Omega_{m, 0}=0.3$ and $\Omega_{\Lambda, 0}=0.7$, are impacted with the inclusion of Mach effect. We will start with $\Omega_{m, 0}=1$, which corresponds to the Einstein de Sitter model. It can be expressed as:

$$
d_{X}=2 R_{X}\left(1-\frac{1}{\sqrt{1+z_{X}}}\right) .
$$

Here $R_{X}=c / H_{X}$ with $H_{X}$ being the Hubble constant corresponding to the expansion model considered. We can use the limit $z=z_{X}=z_{M} \Rightarrow 0$ as the boundary condition for Equation (7) and Equation (8); they both should reduce to the Hubble's law $d=R_{0} z$. Expanding the two equations in the limit yields:

$$
\begin{gathered}
d_{M}\left(t_{0}\right)=R_{M} z_{M}\left(1-\frac{1}{2} z_{M}+\cdots\right), \text { and } \\
d_{X}\left(t_{0}\right)=R_{X} z_{X}\left(1-\frac{3}{4} z_{X}-\cdots\right) .
\end{gathered}
$$

This yields $R_{0} z=R_{M} z_{M}=R_{X} z_{X}$. In the limit, we may write Equation (4) as $z=z_{X}+z_{M}$, or $z=(1-w) z+w z$ where $w$ is the weight factor of Mach effect component and $1-w$ is the weight factor for the expansion component. Comparing these equations with the text book expression for proper distance to a galaxy with redshift $z[10]$ and deceleration parameter $q_{0}$ in the limit $z \Rightarrow 0$ :

$$
d_{P}\left(t_{0}\right)=R_{0} z\left[1-\frac{1}{2}\left(1+q_{0}\right) z\right],
$$

we get for the case of Mach effect $\left(1+q_{0}\right) z / 2=z_{M} / 2=w z / 2$, or $q_{0}=w-1$. And for the case of Einstein de Sitter model $\left(1+q_{0}\right) z / 2=3 z_{X} / 4=3(1-w) z / 4$, or $q_{0}=(1-3 w) / 2$. Equating the two $q_{0}$, we get $w=0.6$ and $q_{0}=-0.4$. The same exercise can be done with Equation (2) with $\Omega_{m, 0} \equiv b$ and $\Omega_{\Lambda, 0} \equiv 1-b$. The equation near $u=0$ may be written as:

$$
\begin{aligned}
d_{X}\left(t_{0}\right) & =R_{X} \int_{0}^{z_{X}}\left[\mathrm{~d} u\left(1-\frac{3}{2} b u+\frac{3}{8} b(9 b-4) u^{2}-\cdots\right)\right] \\
& =\frac{R_{X}}{8} z_{X}\left(8-6 b z_{X}+\cdots\right) \\
& =R_{0} z\left(1-\frac{3}{4} b(1-w) z+\cdots\right)
\end{aligned}
$$

Thus, $1+q_{0}=3 b(1-w) / 2$ for this model. Equating it with $1+q_{0}=w$ for Mach effect, we get $w=1 /\left(1+\frac{2}{3 b}\right)$ and $q_{0}=-1 /\left(1+\frac{3}{2} b\right)$. Noting that for Einstein de Sitter model $b=1$, we get $w=0.6$ and $q_{0}=-0.4$, the same as above. For standard $\Lambda \mathrm{CDM}$ as expansion model, taking $b=0.3$, we get $w=0.31$ and $q_{0}=-0.69$.

The above analysis yields contribution to Hubble constant by the expansion of the universe $H_{X}=0.4 H_{0}$ and by Mach effect $H_{M}=0.6 H_{0}$ when using Einstein de Sitter model for the expansion of the universe, and $H_{X}=0.69 H_{0}$ and 
$H_{M}=0.31 H_{0}$ when using $\Lambda \mathrm{CDM}$ as the expansion model. The data analysis presented in the next section will determine the best parameters.

Let us now see how $z_{X}, z_{M}$ and $z$ are related for all values of $z$, rather than in the limit of $z \ll 0$. Equating $d_{M}$ and $d_{X}$-Equation (7) and Equation (8) - and using Equation (4) we get:

$$
\begin{gathered}
R_{M} \ln \left(1+z_{M}\right)=2 R_{X}\left(1-\frac{1}{\sqrt{1+z_{X}}}\right), \text { or } \\
\frac{R_{0} z}{z_{M}} \ln \left[\frac{1+z}{1+z_{X}}\right]=\frac{2 R_{0} z}{z_{X}}\left(1-\frac{1}{\sqrt{1+z_{X}}}\right), \text { or } \\
\ln \left(\frac{y}{x}\right)=\frac{2(y-x)}{x(x-1)}\left(1-\frac{1}{\sqrt{x}}\right),
\end{gathered}
$$

where for brevity we have substituted $y=1+z$ and $x=1+z_{X}$. This can be numerically solved to determine $x$ for any $y$, and thus $z_{X}$ for any $z$ and also $z_{M}$ for any $z$ since $1+z_{M}=(1+z) /\left(1+z_{X}\right)$. Equation (6), Equation (8), and Equation (13), may now be combined to relate the luminosity distance $d_{L}$ with the observed redshift $z$. As the measured quantity is the distance modulus $\mu$, not the luminosity distance $d_{L}$, we will use the relation:

$$
\begin{gathered}
\mu=5 \log \left(d_{L}\right)+25, \text { or } \\
\mu=5 \log \left(\frac{2 R_{0}(y-1)}{x-1}\left(1-\frac{1}{\sqrt{x}}\right) \sqrt{x y}\right)+1.25 \log (y)+25 .
\end{gathered}
$$

We have used Equation (8) for proper distance in Equation (6). However, we could also use Equation (7) in Equation (6) without any change in the result since Equation (13) has already established equality of the two expressions determining the proper distance.

We could also use Equation (2) for the proper distance in the expanding universe model, equate it to the Mach effect proper distance:

$$
R_{M} \ln \left(1+z_{M}\right)=R_{X} \int_{0}^{z_{X}}\left[\mathrm{~d} u / \sqrt{\left(\Omega_{m, 0}(1+u)^{3}+1-\Omega_{m, 0}\right)}\right],
$$

follow the same exercise as for Equation (13) to relate $z_{X}, z_{M}$ and $z$, and then write the distance modulus relation:

$$
\begin{aligned}
\mu= & 5 \log \left(\frac{R_{0}(y-1)}{x-1} \int_{0}^{x-1}\left[\mathrm{~d} u / \sqrt{\left(\Omega_{m, 0}(1+u)^{3}+1-\Omega_{m, 0}\right)}\right] \sqrt{x y}\right) \\
& +1.25 \log (y)+25
\end{aligned}
$$

\section{Analysis Using Observational $\mu, z$ Database}

The database used in this study is for $580 \mathrm{SNe}$ Ia data points with redshifts $0.015 \leq z \leq 1.414$ as compiled in the Union $2 \mu, z$ database [11] updated to 2017.

We used Matlab curve fitting tool to fit the data using non-linear least square regression. To minimize the impact of large scatter of data points, we applied the "Robust Bi-square" method. This tool fits data by minimizing the summed 
square of the residuals, and reduces the weight of outliers using bisquare weights. The Goodness of Fit is given by parameters SSE (sum of squares due to errors), R-Square and RMSE (root mean square error).

Our objective is to see how well the composite model fits the observational data as compared to the standalone Mach effect model and the expansion models. The results are presented graphically in Figure 1, and numerically in Table 1 ; the table has one extra entry that is discussed below.

The first case is the standard $\Lambda \mathrm{CDM}$ model with two parameter fit, $H_{0}$ and $\Omega_{m}$. The values determined for the parameters are as expected. It provides a good two-parameter fit. This model has the legend " $\Lambda C D M$ " in Figure 1.

In the second case, we have created a composite model by including Mach effect in the first case. The data fit is obtained using Equation (16). The Goodness of Fit numbers have slightly deteriorated compared to the first case. There is a slight decrease in the value of $H_{0}$. However, the matter energy density has become almost 1, the same as for the Einstein de Sitter ("EdeS") model. One could therefore infer that Mach effect is equivalent to cosmological constant!

The third case is for the Einstein de Sitter model. The Goodness of Fit is not good. Also, the Hubble constant value is significantly lower than the previous cases. This model has the legend "EdeS" in Figure 1.

The fourth case is the composite model consisting of the Einstein de Sitter model and the Mach effect. Now the worst case has become the best overall fit case. Hubble constant is slightly lower than for $\Lambda \mathrm{CDM}$ models. This model has the legend "EdeS + Mach" in Figure 1.

The last case considered here is for Mach effect. It presents a not-so-good (OK) one-parameter fit. The Hubble constant value is the highest $\left(H_{0}=70.52\right)$ of all the cases included here. This model has the legend "Mach" in Figure 1.

It may thus be concluded that the one-parameter $\left(H_{0}\right)$ Einstein de Sitter-Mach ("EDSM") composite model is slightly better than the two-parameter

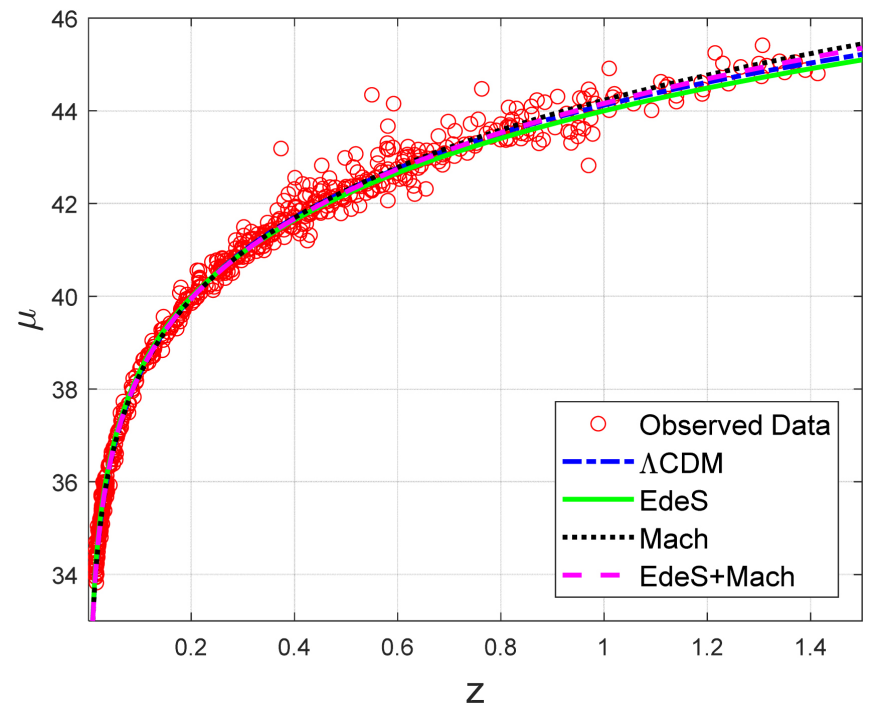

Figure 1. Fitted data curves for four models in Table 1. 
Table 1. Parameters and "Goodness of Fit" for the analysed models, including those whose plots are shown in Figure 1.

\begin{tabular}{ccccccccccccc}
\hline \multirow{2}{*}{ Model } & Parameter & \multicolumn{2}{c}{$95 \%$ Confidence } & Parameter & \multicolumn{2}{c}{$95 \%$ Confidence } & \multicolumn{4}{c}{ Goodness of Fit } \\
\cline { 2 - 12 } & $H_{0}$ & $H_{0}$ Low & $H_{0}$ High & $\Omega_{\mathrm{m}}$ & $\Omega_{\mathrm{m}}$ Low & $\Omega_{\mathrm{m}}$ High & SSE & R-Square & RMSE & Comment \\
\hline$\Lambda$ CDM & $\mathbf{6 9 . 8 5}$ & 70.71 & 69.01 & $\mathbf{0 . 2 8 7 7}$ & 0.2489 & 0.3266 & 24.35 & 0.9959 & 0.2053 & Best 2 parameter fit \\
$\Lambda$ CDM + Mach & $\mathbf{6 9 . 1 4}$ & 70.00 & 68.20 & $\mathbf{0 . 9 9 4 9}$ & 0.8082 & 1.182 & 24.46 & 0.9959 & 0.2057 & Good 2 parameter fit \\
EdeS & 66.30 & 66.82 & 65.72 & 1 & Fixed & Fixed & 28.68 & 0.9951 & 0.2226 & Bad 1 parameter fit \\
EdeS + Mach & $\mathbf{6 9 . 1 1}$ & 69.61 & 68.54 & 1 & Fixed & Fixed & 24.32 & 0.9951 & 0.2049 & Best fit - 1 parameter \\
Mach & 70.52 & 71.04 & 69.93 & NA & NA & NA & 25.54 & 0.9957 & 0.2100 & OK 1 parameter fit \\
\hline
\end{tabular}

$\left(H_{0}, \Omega_{m}\right) \Lambda \mathrm{CDM}$ model for fitting the redshift data while yielding a $1 \%$ lower value of the Hubble constant.

We have shown the $z$ (and hence the time) dependence of the new scale factor $a_{X}$ and other relevant parameter calculated in the EDSM model in Table 2 using Equation (13). It does not involve any fitting of the data, just the model. The conventional scale factor is $1 /(1+z)$. We see that while the two scale factors are the same at $z=0$ and about the same at the lowest non-zero value in the table, $a_{X}$ decreases much more slowly than $1 /(1+z)$ with increasing $z$. This is expected as only a portion $z_{X}$ of $z$ is due to the expansion of the universe, the remaining $z_{M}$ being due to the Mach effect. Figure 2 shows on a log-log grid how the new scale factor $a_{X}$ compares with the conventional scale factor $1 /(1+z)$. The fit to the calculated values is reasonably well represented by $a_{X}(z)=(1+z)^{-0.42}$, especially al lower values of $z$, say up to $z=10$. This may be easier to use in some calculations than numerically solving Equation (13).

The ratio $z_{M} / z_{X}$ is also shown in the table. It increases from the present value of 1.5 to 2.5 for the redshift going from 0 to about 1000 , meaning that Mach effect was contributing $71 \%$ to the redshift then compared to $60 \%$ now.

One question naturally arises: How is the cosmological constant of the $\Lambda \mathrm{CDM}$ model related to the Mach effect of the EDSM model? The question is best answered by analysing the Friedmann equation. The simplest Friedmann equation containing the cosmological constant $\Lambda$ is for the spatially flat universe [10]:

$$
H(t)^{2} \equiv\left(\frac{\dot{a}}{a}\right)^{2}=\frac{8 \pi G}{3 c^{2}} \varepsilon+\frac{\Lambda}{3} .
$$

Here $a$ is the cosmological scale factor, $\dot{a}$ is the time derivative of the scale factor, $G$ is the gravitational constant, and $\varepsilon$ is the energy density. Equation (17) is based on the assumption that all the redshift relates to the velocity of the expansion of the universe: $c z \equiv v(t)=H(t) d(t)$. In the EDSM model, $c z_{X} \equiv v(t)=H_{X}(t) d(t)$, and hence the scale factor that represents the expansion of the universe is really $a_{X}\left[\equiv 1 /\left(1+z_{X}\right)\right]$ with no $\Lambda$. So, we may write the Friedmann equation for the spatially flat universe as:

$$
H_{X}(t)^{2} \equiv\left(\frac{\dot{a}_{X}}{a_{X}}\right)^{2}=\frac{8 \pi G}{3 c^{2}} \varepsilon
$$


Table 2. Parameters based on the numerical solution of Equation (13). The conventional scale factor $a(t)$ is normally taken as $1 /(1+z)$. Here we designate the scale factor as $a_{X}(z)$ since the expansion of the universe is determined by a fraction of the redshift.

\begin{tabular}{cccccc}
\hline$Z$ & $Z_{X}$ & $Z_{M}$ & $1 /(1+z)$ & $a_{X}(z)$ & $Z_{M} / z_{X}$ \\
\hline 0.0000 & 0.0000 & 0.0000 & 1.0000 & 1.0000 & 1.5000 \\
0.014959 & 0.00595 & 0.008956 & 0.985261 & 0.994085 & 1.5052 \\
0.522439 & 0.1847 & 0.285084 & 0.656841 & 0.844096 & 1.543497 \\
1.283658 & 0.3986 & 0.632817 & 0.437894 & 0.715001 & 1.5876 \\
2.425487 & 0.6549 & 1.069906 & 0.291929 & 0.604266 & 1.633694 \\
4.138231 & 0.9621 & 1.618741 & 0.19462 & 0.509658 & 1.682508 \\
6.707347 & 1.3308 & 2.306739 & 0.129746 & 0.429037 & 1.733347 \\
10.56102 & 1.7735 & 3.168386 & 0.086498 & 0.360555 & 1.786516 \\
16.34153 & 2.3055 & 4.246265 & 0.057665 & 0.302526 & 1.841798 \\
25.01229 & 2.9451 & 5.59357 & 0.038443 & 0.253479 & 1.89928 \\
38.01844 & 3.7145 & 7.276263 & 0.025629 & 0.212112 & 1.958881 \\
57.52766 & 4.6405 & 9.376325 & 0.017086 & 0.177289 & 2.020542 \\
86.7915 & 5.7555 & 11.99556 & 0.011391 & 0.148028 & 2.084191 \\
130.6872 & 7.0986 & 15.26049 & 0.007594 & 0.123478 & 2.149789 \\
196.5309 & 8.717 & 19.32838 & 0.005063 & 0.102912 & 2.21732 \\
295.2963 & 10.6679 & 24.39414 & 0.003375 & 0.085705 & 2.286686 \\
443.4444 & 13.0203 & 30.70007 & 0.00225 & 0.071325 & 2.357862 \\
665.6667 & 15.8578 & 38.54648 & 0.0015 & 0.05932 & 2.430758 \\
999 & 19.2814 & 48.30626 & 0.001 & 0.049306 & 2.50533 \\
\hline
\end{tabular}

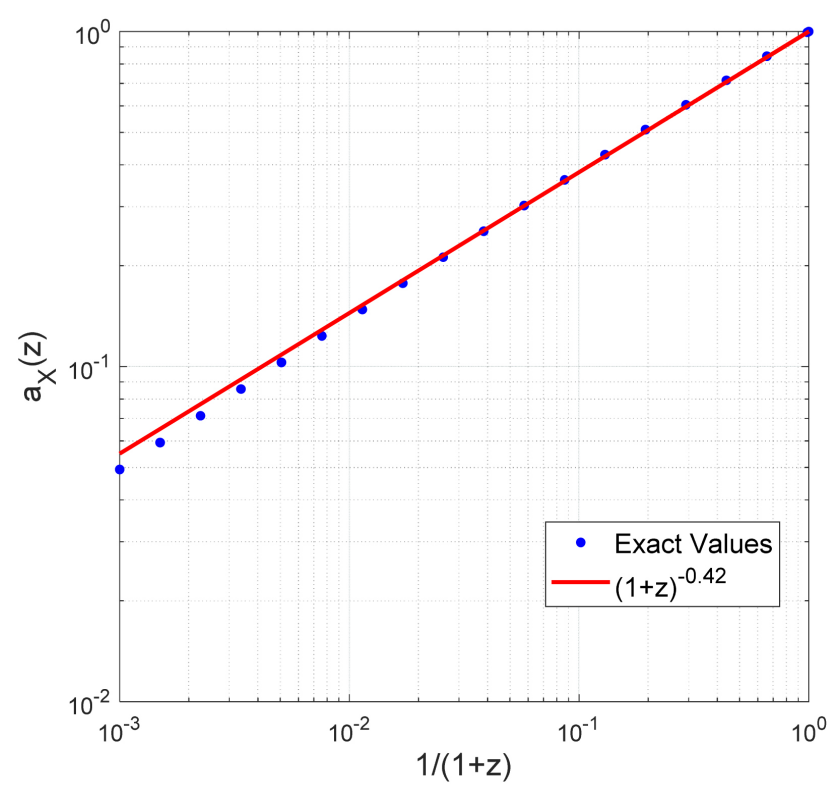

Figure 2. Variation of the new scale factor $a_{X}$ with the conventional scale factor $1 /(1+z)$ plotted on a log-log grid. Calculated values using Equation (13) are shown as blue dots. Also is shown a power fit curve in red. 
Subtracting Equation (18) from Equation (17):

$$
\frac{\Lambda}{3}=H(t)^{2}-H_{X}(t)^{2} .
$$

The right hand side of Equation (19) represents the Mach effect. At $t=t_{0}$ :

$$
\Lambda=3 H_{0}^{2}\left(1-0.4^{2}\right)=2.52 H_{0}^{2}=1.22 \times 10^{-35} \mathrm{~s}^{-2} \text {. }
$$

We have assumed $H_{0}=68 \mathrm{~km} \cdot \mathrm{s}^{-1} \cdot \mathrm{Mpc}^{-1}=2.2 \times 10^{-18} \mathrm{~s}^{-1}$. Dividing by $c^{2}$, $\Lambda=1.36 \times 10^{-52} \mathrm{~m}^{-2}$. In terms of the density parameter, the Mach effect contribution is therefore equivalent to $\Omega_{\Lambda}=0.84$. However, unlike the true cosmological constant, this one is not constant and has time dependence expressed by Equation (19). One could infer that the dark energy is replaced by Mach effect.

Since Equation (18) represents the expansion of the universe, it also determines the critical density rather than Equation (17). It can be easily seen that the new critical density is only $16 \%$ of the generally accepted critical density since $H_{X}=0.4 H_{0}$. And since all the density parameters are expressed relative to the critical density, one could easily see that the baryon density parameter would get a boost by a factor of 6.25. Consequently, the dark matter requirement is drastically reduced.

Another concern: Can the EDSM model explain the time-dilation in type Ia Supernovae and gamma-ray bursts (GRB)? Blondin et al. [12] have shown that the time dilation in Supernovae Ia is proportional to the $(1+z)^{-1}$. This finding is considered by many cosmologist, including López-Corredoira [4] and Orlov and Raikov [5], as inconsistent with other findings, and by Crawford [13], as flawed. Hawkins [14] did not find any time dilation in 878 quasars. Chang [15] studied time dilation in GRB with measured redshift and did not get any conclusive evidence of time dilation. It would be interesting to explore if EDSM model can reconcile such discrepancies as it could explain time dilation proportional to $\left(1+z_{X}\right)^{-1}$, which is significantly less than the time dilation proportional to $(1+z)^{-1}$ of $\Lambda$ CDM model; the smaller time dilation of EDSM model fits well within the error bars in the paper of Blondin et al. [12].

At this early stage of the development of EDSM model, we have not attempted to explain other cosmological phenomena, such as baryonic acoustic oscillations and nucleosynthesis. However, since the EDSM model involves both the Mach effect and the expansion of the universe, the latter due presumably to the big-bang, these phenomena should be possible to account for using the EDSM model albeit with different parameters than the $\Lambda$ CDM model.

Advantages of the EDSM model over $\Lambda$ CDM model:

1) EDSM model needs only one adjustable parameter to fit the data, the Hubble constant, whereas $\Lambda \mathrm{CDM}$ model needs two adjustable parameters; it does not require the cosmological constant.

2) EDSM model keeps the expansion model which is essential for explaining the cosmic microwave background.

3) EDSM model predicts a negative deceleration parameter in compliance 
with observations, albeit a different value than the $\Lambda \mathrm{CDM}$ model.

4) EDSM's Mach effect contribution to the redshift may be deemed equivalent to the cosmological constant in $\Lambda \mathrm{CDM}$ model; there is no need for a controversial constant density parameter that leads to the continuous creation of energy from nothing as universe expands.

5) EDSM model may be considered a simpler model from the viewpoint of Occam's razor having dispensed with ad-hoc assumptions, and dependent only on one verifiable adjustable parameter, the Hubble constant; it does not have conceptual problems associated with the $\Lambda$ CDM model.

6) EDSM model has the potential to explain time dilation claimed to be present in Supernova Ia and some GRB measurements albeit differently than the $\Lambda$ CDM model.

\section{Conclusions}

The extragalactic redshift results not only from the expansion of the universe but also from light losing energy in traveling vast distances from source to observer. By combining the two, through the fact that both the contributions to the redshift measure the same proper distance of the source, we have come up with a one parameter model that fits the redshift SNe Ia data with the same Goodness of Fit as the standard $\Lambda \mathrm{CDM}$ model that requires an additional adjustable parameter. The new model, dubbed EDSM, yields:

1) The Hubble constant $H_{0}=69.11( \pm 0.53) \mathrm{km} / \mathrm{s} / \mathrm{Mpc}$.

2) The deceleration parameter $q_{0}=-0.4$.

3) The $\Lambda$ equivalent for EDSM model $=1.2 \times 10^{-35} \mathrm{~s}^{-2}\left(1.4 \times 10^{-52} \mathrm{~m}^{-2}\right)$, i.e. $\Omega_{\Lambda}=0.84$.

4) The variation of the expansion scale factor with $z$ that is substantially less steeper than $1 /(1+z)$.

5) The Hubble constant component that results from the expansion of the universe is $0.4 H_{0}$ and that from Mach effect is $0.6 H_{0}$.

6) A boost to baryon density parameter by a factor of 6.25 , resulting in a greatly reduced dependence on dark matter to explain cosmology.

\section{Acknowledgements}

I am thankful to Professor Ethan Vishniac and Professor James Peebles for their critical comments on a previous paper that led me to rethink and develop the new approach presented in this paper. I am also thankful to Professor Martin López-Corredoira for his review and suggestions on an early draft of this paper which among other things resulted in the inclusion of $\Lambda \mathrm{CDM}$ models in this study. I am grateful to Dr. Ernst Fischer, Dr. Laszlo Marosi and Dr. David Crawford for sharing their recent research work with me.

\section{Conflicts of Interest}

The authors declare no conflicts of interest regarding the publication of this paper. 


\section{References}

[1] Penzias, A.A. and Wilson, R.W. (1965) A Measurement of Excess Antenna Temperature at $4080 \mathrm{Mc} / \mathrm{s}$. The Astrophysical Journal, 142, 419-421. https://doi.org/10.1086/148307

[2] Lineweaver, C.H. and Barbosa, D. (1998) Cosmic Microwave Background Observations: Implications for Hubble's Constant and the Spectral Parameters N and Q in Cold Dark Matter Critical Density Universes. Astronomy \& Astrophysics, 329, 799-808.

[3] Blanchard, A., Douspis, M., Rowan-Robinson, M. and Sarkar, S. (2003) An Alternative to the Cosmological "Concordance Model". Astronomy \& Astrophysics, 412, 35-44. https://doi.org/10.1051/0004-6361:20031425

[4] López-Corredoira, M. (2017) Test and Problems of the Standard Model in Cosmology. Foundations of Physics, 47, 711-768.

https://doi.org/10.1007/s10701-017-0073-8

[5] Orlov, V.V. and Raikov, A.A. (2016) Cosmological Tests and Evolution of Extragalactic Objects. Astronomy Reports, 60, 477-485. https://doi.org/10.1134/S1063772916030112

[6] Gupta, R.P. (2018) Mass of the Universe and the Redshift. International Journal of Astronomy and Astrophysics, 8, 68-78. https://doi.org/10.4236/ijaa.2018.81005

[7] Poisson, E. (2004) The Motion of Point Particles in Curved Spacetime. Living Reviews in Relativity, 7, 6. https://doi.org/10.12942/lrr-2004-6

[8] Fischer, E. (2007) Redshift from Gravitational Back Reaction. arXivastro-ph/0703791

[9] Peebles, P.J.E. (1993) Principles of Physical Cosmology. Princeton, NJ.

[10] Ryden, B. (2017) Introduction to Cosmology. Cambridge, UK.

[11] Amanullah, R., et al. (2010) Spectra and Hubble Space Telescope Light Curves of Six Type Ia Supernovae at $0.511<\mathrm{z}<1.12$ and the UNION2 Compilation. The Astrophysical Journal, 716, 712-738. https://doi.org/10.1088/0004-637X/716/1/712

[12] Blondin, S., et al. (2008) Time Dilation in Type Ia Supernova Spectra at High Redshift. The Astrophysical Journal, 682, 724-736. https://doi.org/10.1086/589568

[13] Crawford, D.F. (2017) A Problem with the Analysis of Type Ia Supernovae. Open Astronomy, 26, 111-119. https://doi.org/10.1515/astro-2017-0013

[14] Hawkins, M.R.S. (2010) On Time Dilation in Quasar Light Curves. Monthly Notices of the Royal Astronomical Society, 405, 1940-1946. https://doi.org/10.1111/j.1365-2966.2010.16581.x

[15] Chang, H.-Y. (2001) Fourier Analysis of Gamma-Ray Burst Light Curves: Searching for a Direct Signature of Cosmological Time Dilation. The Astrophysical Journal, 557, L85-L88. https://doi.org/10.1086/323331 